\title{
Audio-Haptic Feedback in Mobile Phones
}

\author{
Angela Chang \\ Motorola, Inc. \\ 210 Broadway, $4^{\text {th }} \mathrm{FL}$ \\ Cambridge MA 02139 \\ anjchang@motorola.com
}

\author{
Conor O'Sullivan \\ Motorola, Inc. \\ 600 North U.S. Highway 45, DS-175, \\ Libertyville, IL 60048, USA \\ conor.o'sullivan@motorola.com
}

\begin{abstract}
A new breed of mobile phones has been designed to enable concurrent vibration and audio stimulation, or audiohaptics. This paper aims to share techniques for creating and optimizing audio-haptic effects to enhance the user interface. The authors present audio manipulation techniques specific to the multifunction transducer (MFT) technology. In particular two techniques, the Haptic Inheritance and Synthesis and Matching methods are discussed. These two methods of haptic media generation allow simple creation of vibration content, and also allow for compatibility with non-haptic mobile devices.

The authors present preliminary results of an evaluation of 42 participants comparing audio-based haptic user interface (UI) feedback with audio-only feedback. The results show that users were receptive to audio-haptic UI feedback. The results also suggest that audio-haptics seems to enhance the perception of audio quality.
\end{abstract}

\section{Author Keywords}

Design research, sensorialism, haptics, MFTs, audio, haptic inheritance, synthesis and matching, audio-haptics

\section{ACM Classification Keywords}

H.5.2 [Information Systems]: Information interfaces and presentation (H.5) (I.7)--User Interfaces (D.2.2, H.1.2. I. 3.6) Graphical User Interfaces (GUI); Haptic I/O

\section{INTRODUCTION}

The mobile phone now supports more than just communication (i.e., image creation and web surfing). Predictably, today's phones have more buttons and higher display quality to support the increased applications. In many cases, the physical interaction spaces of the interface have largely remained the same. Users are still confined to buttons, keypads and small screens to manage content.

Because of the increased amount of information that passes through these interfaces, the screen space and button space can become cluttered and hard to read. There are many suggested solutions, most notably the development of new interaction techniques (suggested by new form factors and techniques $[4,6])$.

One proposed solution is the use of sensorialism[8] in the

Copyright is held by the author/owner(s).

CHI 2005, April 2-7, 2005, Portland, Oregon, USA.

ACM 1-59593-002-7/05/0004.

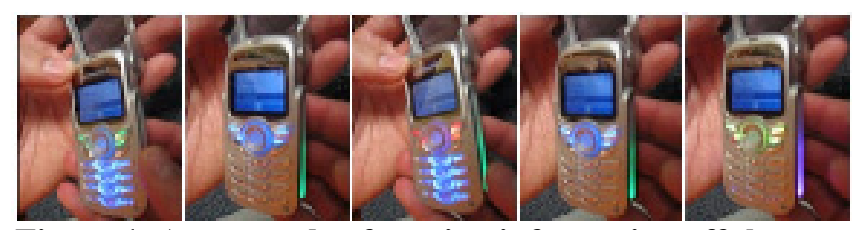

Figure 1. An example of moving information off the screen space. The e380 phone has tri-color sidebands and vibration to light up and shake depending on ringtone.

device to offload information onto other physical spaces and modalities[2,3], e.g. by displaying information on the sidebars or through vibration [1,7] as in Figure 1. By integrated stimulation of the five basic senses: touch, taste, hearing, sight and smell, the sense of cognition is engaged more fully. The resulting sensory integration also allows a richer emotional experience for the user.

Offloading more information to the haptic modality was proposed. Commercially, tactile sensations are separate from user interface sounds. Tactile sensations already exist in vibrating ringtones and vibrating ring alerts. A logical next step would be integration of vibration content into the user interface.

\section{HAPTIC ENABLERS}

There are currently three solutions for presenting vibration in mobile devices. The most popular solution is the rotary mass vibrator (RMV) or pager motor, which allows on-off vibration. Another solution is slim piezoelectric actuators requiring high voltage. The next solution is the MultiFunction Transducer, (MFT), a speaker which can produce both audible and vibrotactile output from an audio signal.

The MFTs were favored for many reasons (size, power efficiency, least lag time). Another important feature is the effect of the synchronicity of sound with vibration, audiohaptics. This significance of this effect is that, if designed correctly, special audio files can be played on both MFTenabled and non-haptic phones. The content creator can save time by creating one set of UI sounds and ringtone media for both types of phones.

In phones with MFTs, such as the Motorola e380, e398, e680, haptic effects can be felt when audio frequencies are between approximately $100-300 \mathrm{~Hz}$. Frequencies above $300 \mathrm{~Hz}$ are heard as audio. By manipulating different parameters (e.g frequency, amplitude, and waveform), it is possible to design a variety of sensations that can range from a subtle buzz to discrete taps. 


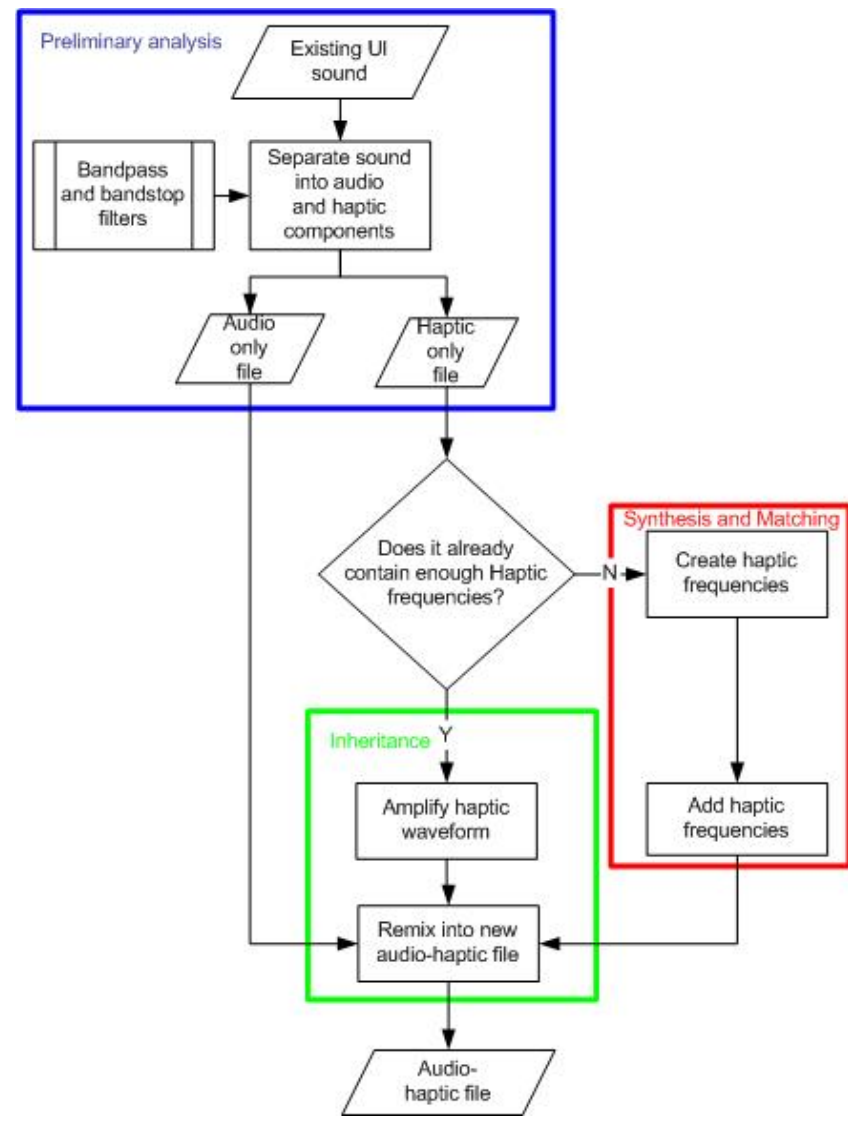

Figure 2. Flow diagram for 'haptic inheritance' and 'synthesis and matching' techniques to create audio-haptic media.

\section{METHODS FOR HAPTIC MEDIA GENERATION}

There are two methods offered for processing sound to optimize the effect of haptic sensation, using actuators such as the MFT which can vibrate at low frequencies. The proposed flow diagram for creating audio-haptics is depicted in Figure 2.

Both methods require a preliminary analysis of the sound and its components to determine the appropriate path for generation of the haptic effect.

The first method describes a technique the authors refer to as 'haptic inheritance'. This method is based on the authors' premise that the sound itself contains enough inherent haptic information that this method of processing can exploit to deliver a pleasing and appropriate tactile or haptic icon. The second method uses some of the procedures described above and is referred to here as 'synthesis and matching'. The second method enables the designer to approximate a desired response by adding extra haptic frequencies, perhaps from sounds of a haptic library.

\section{Haptic Inheritance}

This method assumes some relevant degree of audio information around the resonant peak of the audio-response actuator. The important part of the process to resolve using this method is to split the sound into its constituent parts, that of haptic and audio. Of course it is useful to point out that these parts both contain audio (and similarly haptic) information. However this method is concerned specifically with the optimization for an audio-response actuator on a narrow-bandwidth output device, thereby limiting the need for concern about crossover in regard to these bins.

One such technique for arriving at these constituent bins is given here. Two filters can be applied to the original sound signal so that these bins are obtained and further processing can take place. The first, a band stop filter, is used to isolate the audio portion of the source signal. The authors here employ a band stop filter with a low cutoff at $129 \mathrm{~Hz}$ and high cutoff at $177 \mathrm{~Hz}$. These cutoff frequency values are chosen relative to the resonant peak of the audio-response actuator. To obtain the second bin, a converse type of filter is used, here a band-pass filter.

Once these constituent bins are obtained, it is then necessary to process them efficiently for the optimal level of haptic and audio re-combination output. In the first instance of this step of the method, the haptic bin is analyzed to determine the level of existing tactile effect.

The best way to perform this analysis is of course through a physical determination of sensation, however an analysis and subsequent algorithmic adjustment of the signal is given here and has been found to be both useful and compelling. The amplification of this haptic bin is given by a normalization of up to $-12 \mathrm{dBV}$ rms.

The second part of this step involves additional filtering of the audio bin to further optimize for the audio speaker part of the output device. For a typical device used for this study, the frequency response of the output device begins to drop off sharply from $900 \mathrm{~Hz}$ through $400 \mathrm{~Hz}$ and below. The frequencies of the audio bin are thus ramped off accordingly.

\section{Synthesis and Matching}

This technique is best used when an analysis of the input signal reveals no useful audio information around the resonant ' $Q$ ' peak of the audio-response actuator. The method involves a more subjective design approach. It incorporates the generation of haptic textures using several possible methods and a means of matching the desired haptic response to appropriate or preferred points in the audio/time signal.

Haptic icons are information signals that occur in the low frequency range between $20-300 \mathrm{~Hz}$, where the skin's sensitivity to vibration is engaged. These haptic icons are designed to serve as concise tactile representations of information to the user, similar to audio and visual icons, (i.e. messaging, alert, and confirmation). Some means to generate haptic texture icons include standard audio synthesis techniques such as modulation, mixing, filtering and enveloping. Alternatively, an approach suggested by the authors is to derive a haptic signal using the Haptic Inheritance method described above from one signal and incorporate this into the design of another. The techniques for synthesis and manipulation of audio signals are the 
same as can be found in any comprehensive computer music textbook [5] and so need not be described here.

There are a couple of points for the designer of haptic textures to note however; work should be performed in the frequency domain around the resonant Q peak of the MFT and also the same normalization of the haptic signal should apply. This normalization is necessary due to hardware drive limitations.

\section{HAPTIC EVALUATIONS}

We had people compare a haptic phone with a non-haptic phone at DIS 2004. Users were asked to try navigating the menu keys and then play ring tones to compare the two phones. They were allowed to test out the two phones in random order, and were allowed to compare the phones side by side. Then they were asked to fill out a questionnaire (figure 3) which assessed whether people could distinguish the haptics, and which phone experience they preferred. There was also ample space for comments on their perception of the UI.

We used a commercially available e398 phone with dual MFTs. As shown in the top of figure 4a, the e398 phone was loaded with audio-haptic ringtones and UI-sounds for the menu key, power up key, and send and end keys, generated in the manner described above. The other phone, a Motorola v400 (in figure 4b, was clam shaped) and was commercially available at the time of the test. It contained a RMV, and the standard audio files.

During the conference, 42 volunteers ( 30 male, 12 female) tried out the phones for approximately 10 minutes each. The average age group of each user was between 21-35.

\section{Results}

Tables 1-3 (at right) show the results of the questionnaires collected (with the largest number responses highlighted).

\section{Experience feedback form}

Age range: $<13$ 14-20 21-35 36-50 50+

Gender: Male Female

Can you tell the difference between the user interface buttons on the two phones? If so, please describe the difference.

Can you feel any sensations when pressing the buttons?

Rate the sensations: 1(dislike) 2345 (like)

Which phone feels better? Why?

Which phone sounds better? Why?

Comments: please provide any general comments.

Figure 3. After performing the comparison task, participants were asked to fill out the above questionnaire. 4a
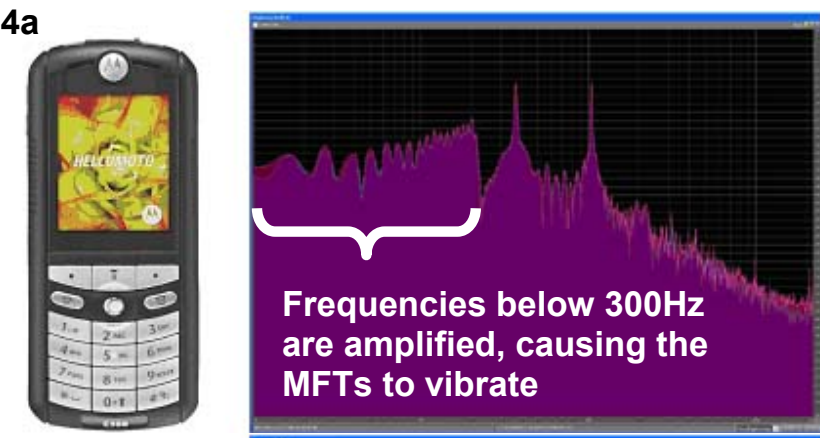

Frequencies below $300 \mathrm{~Hz}$

are amplified, causing the

MFTs to vibrate

4b
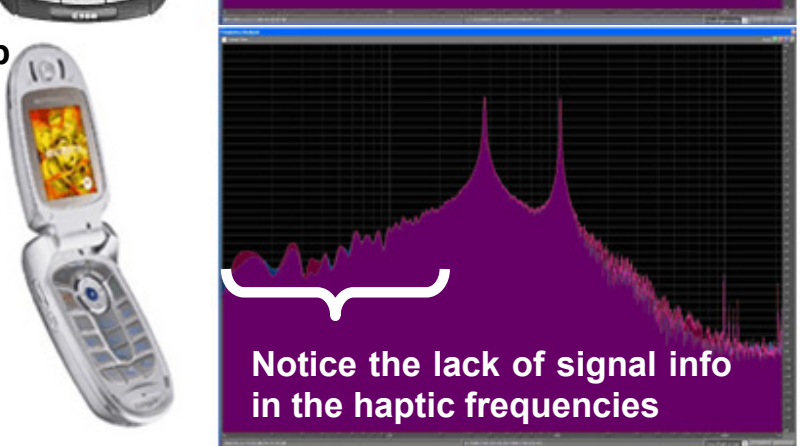

Figure 4. An e398 phone with audio-haptic sounds (4a) was compared to a $\mathbf{v} 400$ phone with audio-only sounds (4b). When playing an audio-haptic sound, the e398 can shake in response to vibration due to its embedded

MFTs. Spectrograms of a UI sound file show the amplification of haptic frequencies in the MFT-enabled phone.

Table 1. Select which phone had a better feel.

\begin{tabular}{|l|l|}
\hline \multicolumn{2}{|l|}{ Which phone had a better feel? Pick one: } \\
\hline haptic & 35 \\
\hline same or none & 5 \\
\hline clam & 2 \\
\hline
\end{tabular}

Table 2. Select which phone has better audio.

\begin{tabular}{|l|l|}
\hline \multicolumn{2}{|l|}{ Which phone had better audio? Pick one: } \\
\hline haptic & 20 \\
\hline same or none & 22 \\
\hline clam & 0 \\
\hline
\end{tabular}

Table 3. Rate the sensations from 1(dislike) to 5 (like).

\begin{tabular}{|l|l|}
\hline \multicolumn{2}{|l|}{ Rate your affinity for the haptic vibrations: } \\
\hline 1 (dislike) & 0 \\
\hline 2 & 4 \\
\hline 3 & 21 \\
\hline 4 & 6 \\
\hline 5 (like) & 11 \\
\hline
\end{tabular}




\section{Observations}

On a scale of 1 to 5 , with 5 being the most agreeable, the haptic sensations on the 398 phone received a mean rating of $3.92+/-0.89$ out of 5 . This "above average" rating is an important result, as it shows that most people said that they liked the haptic feedback phone.

When comparing the user interface feel of the two phones, there was no doubt which phone felt better. 35 out of 42 people reported that the haptic phone felt better. One of the respondents reported liking the clam phone better said he was responding to the form factor and not the UI sounds.

20 participants thought the haptic phone had better audio, while 22 felt the audio between the two were the same. One of the reasons why more people might not have been able to judge the audio well was that the conference room was quite noisy. None of the participants reported that the nonhaptic phone sounded better. Next, we ran a Pearson correlation analysis between the sound and feel responses.

We found that there was a significant relation between sensation and the perception of sound quality. Phones that are said to 'sound better' are significantly correlated with phones that garner high 'sensations' ratings. This is a nice result, but not totally surprising. Phones that are said to 'sound better' are significantly correlated with phones that garner high 'sensations' ratings.

We attribute this occurrence to the idea that phones that sound good also rate high in sensation. The presence of haptics increases the perception of sound in the phone. We can infer that there is some correlation between sound quality and haptic sensation.

One user reported that they liked the haptic vibrations, but were confused by the shape differences between phones. We had decided to use the different shaped phones because we felt the audio and UI comparison among the two phones would reflect the user experience on commercially available hardware. A future comparison should use two identical phones with haptic media enabled on only one phone. Another user reported that although she liked the vibrations, they could get annoying or the user might become desensitized if used for every click or selection.

Some other the comments we received were "many possibilities for vibration as a medium", "interesting-- need more variety of sensations" and "The little haptic feedback on the button press is good. The big wiggles feel strange though." In general, users were very positive about the possibility of expanding the user interface to include more variations in vibration.

\section{CONCLUSION}

Methods of design for audio-haptics and its application to the MFT audio-response actuator were presented. The resulting waveforms result in a consistent feel for haptics, with minimal audio distortion. The filters presented can allow real-time creation of haptic effects. As a result, this tactile enhancement of audio signals forms a richer set of interface experiences for the user.

Furthermore, the methods described here allow content creators to easily incorporate haptics into the UI or ringtones. Audio-haptic media can also be played with reasonable quality on phones without haptic capabilities. This is important for backward compatibility and also for simplifying the work of the UI content creator. Furthermore, the use of audio to drive vibration allows other applications to access the haptic channel by simply playing audio. This feature opens up the possibility of adding haptic feedback to mobile games without the need for extra software control.

The authors have here presented the results of preliminary investigations into the incorporation of this type of haptic feedback into the mobile user interface. The authors feel satisfied at the degree of positive feedback this work received and agree that further contributions are necessary to refine the design space of haptic icons, human factors testing, and exploration of audio-haptic applications.

\section{ACKNOWLEDGMENTS}

We thank the members of Motorola Consumer Experience Design for their generous support of this haptics work. In particular, we would like to thank the members of the audio team, the human factors team, and the MFT phone product teams. Finally, we would like to thank all the volunteers at DIS who served as test subjects for our preliminary evaluation.

\section{REFERENCES}

1. Brewster, S. and Brown, L. Demonstrations: Non-visual information display using tactons, Ext. Abstracts CHI 2004, ACM Press, 787-788.

2. Ishii, H. and Ullmer, B. Emerging frameworks for tangible user interfaces, IBM Systems Journal 39 , (3\&4): 915- (2000).

3. Les Nelson, et.al. Quiet Calls: Talking Silently on Mobile Phones, Proc. CHI 2001, ACM Press, 174-181.

4. Michelitsch, G., Williams, J., Osen, M., Jiminez, B., Rapp, S. Haptic chameleon: a new concept of shapechanging user interface controls with force feedback, Ext. Abstracts CHI 2004, ACM Press, 1305-1308.

5. Roads, C. The Computer Music Tutorial, MIT Press, Feb 1996.

6. Schwesig, C, Poupyrev, I., Mori, E., Gummi: a Bendable Computer, Proc. CHI 2004, ACM Press, 263270.

7. Tan, H. Z., Perceptual user interfaces: haptic interfaces; Communications of the ACM 43, 3 (Mar. 2000), $40-$ 41.

8. Tollmar, K., Junestrand, S., Torgny, O., Virtually Living Together, Proc. DIS 2000, ACM Press (2001), 83-91. 